\title{
Adaptive Fuzzy PID in the Application of the Furnace Temperature Control System
}

\author{
Zhengqiang Guan ${ }^{1, a}$, Xiaoming Luo ${ }^{2, b}$, Lepeng Song ${ }^{1, c}$ \\ ${ }^{1}$ Chongqing University of science and technology, Chongqing, China \\ bsjkgzq@126.com, ${ }^{\mathrm{c}}$ slphq@163.com \\ ${ }^{2}$ Property management co., Itd. Shengzhen Merchants., Shengzhen city, Guangdong, China \\ a70luo@163.com
}

Keywords: Simulation; Temperature control; Self-tuning fuzzy PID

\begin{abstract}
According to the set temperature, the parameter self-tuning fuzzy PID control algorithm is used furnace temperature automatic control and fuzzy PID control process to make use of the MATLAB simulation. Simulation results show that compared with conventional PID control, the parameter self-tuning fuzzy PID control algorithm improves the system response, shorten the adjusting time, enhances the disruptive, demonstrate the superiority of the fuzzy PID.
\end{abstract}

\section{Introduction}

With conventional PID controller can realize the system structure is simple, easy to implement, robust, etc, but in the process of the practical work of interference, the influence of nonlinear factors, such as the furnace temperature control system with pure lag, nonlinear and parameter time-varying characteristics, its control effect is poor and use the fuzzy PID controller to control the furnace temperature[1-3], it can improve the response speed, eliminate the steady-state error, and a satisfactory control of complex system, so as to achieve control requirements and fuzzy PID control algorithm to automatic control of liquid flow, reduced small overshoot, the system shortens the time of adjustment to keep fertilizer rate more effectively in a given range[4] [5]. In the variable frequency speed control using fuzzy PID control algorithm is greatly accelerated the response speed of system, using inverter control of PLC analog output control belt motor speed, so as to achieve the goal of controlling material flow[6] [7]. A fuzzy PID parameters self-tuning system used in furnace temperature control, through experiment and simulation is superior to the conventional PID control of furnace temperature results are obtained.

\section{The transfer function of controlled object}

Controlled object is electric furnace, its structure is shown in figure 1:

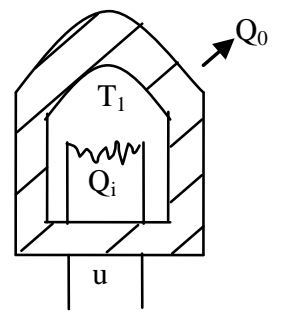

Figure 1 Electric furnace section

According to the thermal knowledge, the dynamic equation of electric furnace is for

$$
M C \frac{d\left(T_{I}-T_{0}\right)}{d t}+H A\left(T_{1}-T_{0}\right)=Q_{i}
$$

The meaning of the parameter is (1). M - Heating wire quality. C - Specific heat; T1 - current temperature; T0 - equilibrium temperature; $\mathrm{H}$ - conduction coefficient; $\mathrm{A}$ - heat transfer area; $\mathrm{Q}_{\mathrm{i}}$ - the current temperature. 
Due to Qi and the ratio of the square of the applied voltage, so the Qi and a nonlinear relationship with $u$ can be in balance $(\mathrm{Q} 0, \mathrm{u} 0)$ linearization near get.

$$
K_{u}=\frac{\Delta Q_{i}}{\Delta u}
$$

According to (1) and (2) electric furnace can be obtained incremental differential equation

$$
T \frac{d \Delta T}{d t}+\Delta T=k \Delta u
$$

The meaning of the parameter in (3)

$\mathrm{T}$ - time constant;

$$
T=\frac{M C}{H A}
$$

$\mathrm{K}$ - conduction coefficient;

$$
k=\frac{K_{u}}{H A}
$$

The (3) after the Laplace transform to get

$$
T s \Delta T(s)+\Delta T(s)=k \Delta U(s)
$$

Electric furnace are obtained by (6), under the condition of zero initial transfer function for

$$
G(s)=\frac{\Delta T(s)}{\Delta U(s)}=\frac{k}{T s+1}
$$

\section{Parameters self-tuning fuzzy PID temperature control system design}

The conventional fuzzy controller structure diagram as shown in figure 2.Input real value to the system, first ofall by fuzzy processing and output aqualified semantic fuzzy membership degree of the set (total between 0, 1), for each fuzzy rules, the degree of each part to be fulfilled in the premise, then these approved by membershipdegree of fuzzy output to the output fuzzy operator to deal with a single real value, this value estimate says theprerequisite for the rule of the output. The fuzzy rules are represented as "if... then..."Conditional statements, itsessence is expressed as the premise of changes in multiple conditions produce a decision results by reasoning. Finally once for each rule specifies theappropriate rights, it implements the implication, the input of the process of fuzzy implication is the premise of a given rule of a single value, while the output is a fuzzy set, the fuzzy set by the clustering to form a single fuzzy set. The fuzzy set of fuzzy by processing final output a true value.

Parameter adaptive fuzzy PID controller design idea is: first, find out the three PID control parameters and the system error e and the deviation rate of ec fuzzy relation, and then with the help of fuzzy control method, three parameters of PID controller online modification, the experiment in order to achieve the purpose, its structure is shown in figure 3. 


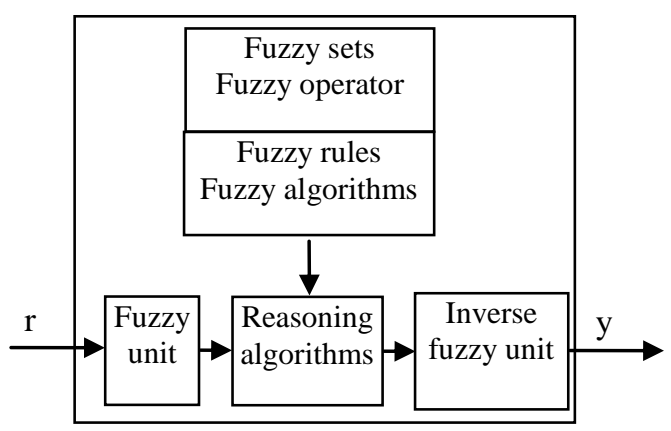

Figure2 The structure of fuzzy controller

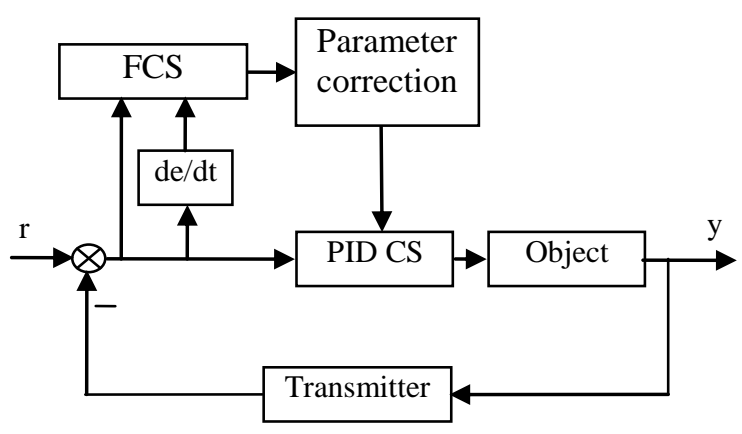

Figure3 Parameter adaptive fuzzy PID controller

\section{Digital PID control algorithm}

The PID control law for

$$
u_{t}=K_{P}\left[e(t)+\frac{1}{T_{I}} \int_{0}^{t} e(t)+T_{D} \frac{d e(t)}{d t}\right]
$$

Type of e ( $t$ ) - deviation control system; $u_{t}$ - the output of the PID controller; $K_{P}$ - ratio; $\mathrm{T}_{\mathrm{I}}$ - the integral time constant; $\mathrm{T}_{\mathrm{D}}$ - differential time constant. To discretization (8), we can get

$$
u_{k}=K_{P}\left\{e(k)+\frac{T}{T_{I}} \sum_{j=0}^{k} e(j)+\frac{T_{D}}{T}[e(k)-e(k-1)]\right\}
$$

Type of $\mathrm{T}$ - sampling period; $\mathrm{K}$ - sampling sequence number.

\section{Variable selection and Optimization}

In practice, because the stand or fall of quantization factor and scaling factor on the system has a great relationship, so their choice is a very critical problem. Fuzzy controller input for e and de/dt, parameters for $K_{P}, K_{D}$, and alpha and input is accurate quantity, so must first carries on the fuzzy processing. Quantitative factors of input e and de/dt is $\mathrm{K}_{1}$ and $\mathrm{K}_{2}$, respectively, and the parameters $\mathrm{K}_{\mathrm{P}}$ ', $\mathrm{K}_{\mathrm{D}}$ ' and alpha 'corresponding control quantity is $\mathrm{K}_{3}$ respectively, and the scaling factor $\mathrm{K}_{4}$ and $\mathrm{K}_{5}$. Theory of fuzzy controller of two input domain is $0 \sim 1$, fuzzy subsets are NB, NM, NS, ZO, PS, $\mathrm{PM}$ and $\mathrm{PB}$, parameters $\mathrm{K}_{\mathrm{P}}$, $\mathrm{K}_{\mathrm{D}}$ ' theory of domain is $0 \sim 1$, fuzzy subsets of $\mathrm{S}$ and $\mathrm{B}$, the theory of domain is $2,3,4,5$. In addition to the alpha using a single point of membership functions, the rest adopt symmetric triangular function.

\section{The fuzzy control rule base design}

Fuzzy controller with two input output model, according to the PID parameters influence on furnace temperature and the principle of parameter setting, can be concluded that in view of the KP, $\mathrm{KI}, \mathrm{KD}$ respectively setting control rules table, using the fuzzy rule statements in the form of "if e is $\mathrm{A}$ and ec is $\mathrm{B}$, then $\mathrm{KP}$ ' is $\mathrm{C}$ and $\mathrm{KD}$ ' is $\mathrm{D}$ and $\alpha$ is E.", among them $\mathrm{A}, \mathrm{B}, \mathrm{C}, \mathrm{D}, \mathrm{E}$, both said the corresponding parameters of fuzzy sets. Fuzzy control table of $K_{P}$ can be obtained as shown in Table 1, Fuzzy control table of $K_{D}$ can be obtained as shown in Table 2, Fuzzy control table of acan be obtained as shown in Table 3.

\section{System simulation and Analysis}

Using MATLAB, Simulink and Fuzzy tools respectively is to be established conventional PID control and fuzzy PID control of furnace temperature control system simulation. Simulation system block diagram as shown in the figure Figure4 and Figure5. Figure 6 shows the results of Simulation curve. 
Table 1 Fuzzy control table of $\mathrm{K}_{\mathrm{P}}$

\begin{tabular}{|c|c|c|c|c|c|c|c|c|}
\hline \multirow{2}{*}{$\mathrm{K}_{\mathrm{D}}$} & & \multicolumn{7}{|c|}{$\mathrm{ec}$} \\
\hline & & $\mathrm{NB}$ & NM & NS & $\mathrm{ZO}$ & PS & PM & PB \\
\hline \multirow{7}{*}{$\mathrm{e}$} & NB & S & S & $S$ & $S$ & $S$ & $\mathrm{~S}$ & $\mathrm{~S}$ \\
\hline & NM & B & B & $S$ & S & $S$ & B & B \\
\hline & NS & B & B & B & S & B & B & B \\
\hline & $\mathrm{ZO}$ & B & B & B & B & B & B & B \\
\hline & PS & B & B & B & $S$ & B & B & B \\
\hline & PM & B & B & $S$ & S & S & B & B \\
\hline & PB & $S$ & $S$ & $S$ & $S$ & $S$ & $S$ & $S$ \\
\hline
\end{tabular}

Table 3 Fuzzy control table of $\alpha$

\begin{tabular}{|c|c|c|c|c|c|c|c|c|}
\hline \multirow{2}{*}{$\alpha$} & & \multicolumn{7}{|c|}{$\mathrm{ec}$} \\
\hline & & $\mathrm{NB}$ & NM & $\mathrm{NS}$ & $\mathrm{ZO}$ & PS & PM & $\mathrm{PB}$ \\
\hline \multirow{7}{*}{$\mathrm{e}$} & NB & 2 & 2 & 2 & 2 & 2 & 2 & 2 \\
\hline & $\mathrm{NM}$ & 3 & 3 & 2 & 2 & 2 & 3 & 3 \\
\hline & NS & 4 & 3 & 3 & 2 & 3 & 3 & 4 \\
\hline & $\mathrm{ZO}$ & 5 & 4 & 3 & 3 & 3 & 4 & 5 \\
\hline & PS & 4 & 3 & 3 & 2 & 3 & 3 & 4 \\
\hline & PM & 3 & 3 & 2 & 2 & 2 & 3 & 3 \\
\hline & PB & 2 & 2 & 2 & 2 & 2 & 2 & 2 \\
\hline
\end{tabular}

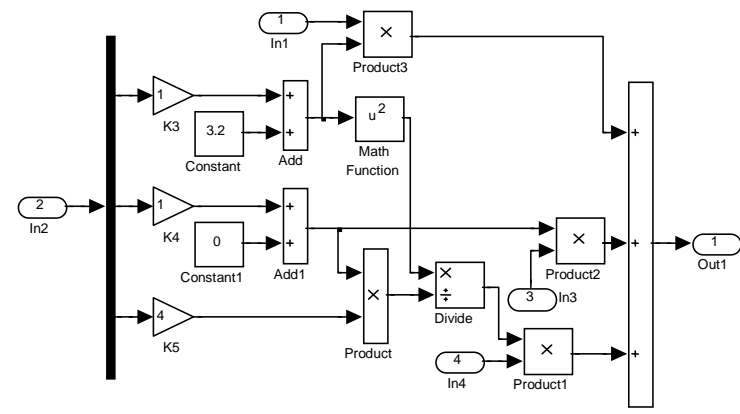

Figure5 Subsystem block diagram
Table 2 Fuzzy control table of $\mathrm{K}_{\mathrm{D}}$

\begin{tabular}{|c|c|c|c|c|c|c|c|c|}
\hline \multirow{2}{*}{$\mathrm{K}_{\mathrm{P}}$} & & \multicolumn{7}{|c|}{ ec } \\
\hline & & NB & $\mathrm{NM}$ & NS & $\mathrm{ZO}$ & PS & PM & PB \\
\hline \multirow{7}{*}{$\mathrm{e}$} & NB & $\mathrm{B}$ & $\mathrm{B}$ & $\mathrm{B}$ & B & B & $\mathrm{B}$ & B \\
\hline & NM & $S$ & B & B & B & B & B & B \\
\hline & NS & $S$ & S & B & B & B & S & $S$ \\
\hline & $\mathrm{ZO}$ & $S$ & $S$ & $S$ & B & $S$ & $S$ & $S$ \\
\hline & PS & $S$ & $S$ & B & B & B & $S$ & $S$ \\
\hline & PM & $S$ & B & B & B & B & B & $S$ \\
\hline & PB & B & B & B & B & B & B & B \\
\hline
\end{tabular}

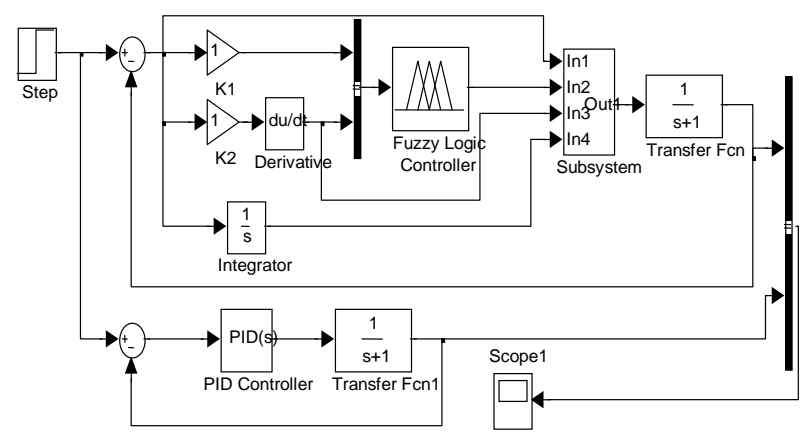

Figure4 Simulation system block diagram

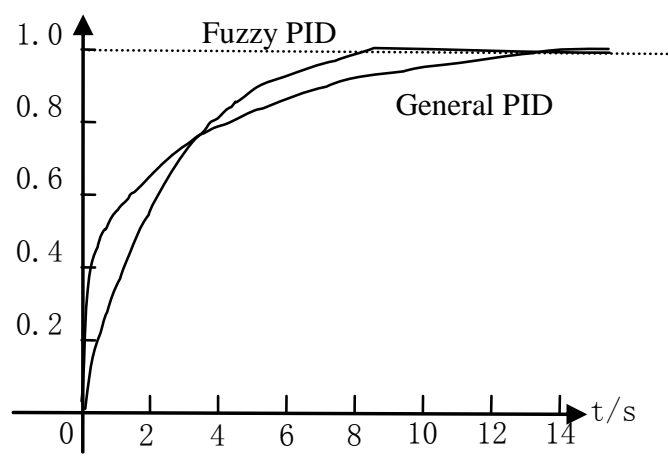

Figure6 Results of Simulation curve

\section{Conclusion}

From the simulation results we can see the parameter self-tuning fuzzy PID control is applied to the furnace temperature control system, can make the system performance index is increased significantly, illustrate the system uses fuzzy PID control method is effective and advantageous.

\section{Acknowledgements (Project Funding)}

This research is funded by Research Foundation of Chongqing University of Science \& Technology, the project No. is CK2011Z16 and CK2013B15.

This research is funded by Research Foundation of Chongqing Education Committee, the project No. is KJ131416 and KJ133501.

This research is funded by Research Foundation of The Natural Foundation of Chongqing City, the project No. is cstc2014jcyjA70001.

The work described in this paper was supported by the Application development projects of CQ CSTC under Grant No. cstc2014yykfA80012.

Corresponding Author: Lepeng Song, Chongqing University of science and technology, School of Electrical and Information Engineering, 401331, Chongqing, China. 


\section{References}

[1] Lin Li, Mengxiong Zeng. Application of Fuzzy PID Control in Motion Control. Mechanical and electronic, 2006(2): 65-67.

[2] Mudan Li, Lihong Li, Zhangwei Lei. Batching Scale Based on Fuzzy PID Control System. Test and technology of China, 2008(02): 116-119.

[3] Chunlan 1v, Liguo Wang, Yanan Meng, Delong Jiang. Based on the Fuzzy Self-tuning PID Parameters of the Controller Design. Journal of jilin institute of chemical industry, 2002, 19(2): 33-35.

[4] Chang Guo, Qinwei Gao, Guimei Cui. New Applications of Fuzzy PID Control Algorithm in Frequency Control of Motor Speed. Motor and control applications, 2011, 38(8): 19-22.

[5] Ziwen Dong, Zhangqing Zhu. Fuzzy PID Control in the Application of Double Closed-loop Speed Regulating System Research. Electrical drive and automatic control, 2010, 32(5): 14-16.

[6] Yan Li, Xiaoming Du. The Application of Fuzzy PID Control in Greenhouse Environment. Agricultural mechanization research, 2010(08): 163-177.

[7] Deqi Ren, Bin Guo. Fuzzy PID Parameters Self-tuning in the Application of the Furnace Temperature Control System. Electrical applications, 2008, 20(19): 77-79. 